\title{
Spipubstsaúde
}

ARTIGO ORIGINAL

\section{Pesquisa de resíduos de antibióticos em leite in natura, pasteurizado e UHT}

\section{Research of antibiotic in milk in natura, pasteurized and UHT}

\section{Ana Beatriz Melo Guimarães ${ }^{1 \oplus}$, Márcia Facundo Aragão ${ }^{1 \oplus}$, Renata Albuquerque $\operatorname{Costa}^{16}$, José Rodolfo Teixeira da Cunha ${ }^{2}$, Thaiane Vasconcelos Carvalho ${ }^{1}$}

${ }^{1}$ Centro Universitário INTA - UNINTA. Sobral-CE, Brasil. Universidade Federal do Maranhão, São Luís-MA, Brasil.

E-mail: anabeatrizmelog@gmail.com

Como citar: Guimarães, A.B.M., Aragão, M.F., Costa, R.A., Cunha, J.R.T., Carvalho, T.V., 2019. Pesquisa de resíduos de antibióticos em leite in natura, pasteurizado e UHT, 2, a012. DOI: https://dx.doi.org/10.31533/pubsaude2.a 012

Recebido: 01 out. 2019.

Revisado e aceito: 28 out. 2019.

Conflito de interesse: os autores declaram, em relação aos produtos e companhias descritos nesse artigo, não ter interesses associativos, comerciais, de propriedade ou financeiros que representem conflito de interesse.

Licenciamento: Este artigo é publicado na modalidade Acesso Aberto sob a licença Creative Commons Atribuição 4.0 (CC-BY 4.0).
R e s u m o. A presença de resíduos de antibióticos no leite pode ocorrer devido sua adição proposital na alimentação do animal e no produto final ou, devido ao não respeito ao período de latência após administração desses medicamentos em animais leiteiros, levando assim reações indesejáveis ao ser humano, como por exemplo, a hipersensibilidade, resistência bacteriana e desequilíbrio da microbiota intestinal. Desta forma, o objetivo da pesquisa foi investigar a presença de resíduos de antibióticos em amostras de leite in natura, pasteurizado e UHT comercializadas nos municípios de Bela Cruz e Sobral, localizadas no Ceará. Foram analisadas 90 amostras de leite, das quais 30 foram de leite in natura, 30 leites pasteurizados e 30 leites UHT. Foram realizadas análises qualitativas de detecção de resíduo de antibióticos em leite seguindo as instruções presentes no kit Cow Side II Test. Das 90 amostras estudadas, 63,33\% mostraram-se positivas a presença de antibióticos, onde 70\% das amostras de leite in natura, 73,33\% das pasteurizadas e $46,67 \%$ das amostras de leite UHT apresentaram reação positiva a presença de antibióticos. Concluiu-se que na maioria das amostras estudadas houve a presença de resíduos de antibióticos e, não foi realizada a quantificação de resíduos presentes para comparar com o limite aceitável devido o kit utilizado para os testes ser de caráter qualitativo. Deve-se destacar também que apesar da não quantificação, a presença desses resíduos ainda deve ser considerada uma preocupação para os consumidores, pois sabe-se que podem causar reações alérgicas em indivíduos com hipersensibilidade, além de estarem expondo os consumidores a outros riscos graves.

P a l a v r a s - c h a v e : antibióticos, leite, resistência. 
A b stract. The presence of antibiotic residues in milk may occur due to their deliberate addition to the animal's feed and the final product or, due to non-compliance with the latency period after administration of these drugs in dairy animals, thus leading to undesirable reactions to humans, such as hypersensitivity, bacterial resistance and imbalance of the intestinal microbiota. Thus, the objective of the research was to investigate the presence of antibiotic residues in fresh, pasteurized and UHT milk samples marketed in the cities of Bela Cruz and Sobral, located in Ceará. Ninety milk samples were analyzed, of which 30 were fresh milk, 30 pasteurized milk and 30 UHT milk. Qualitative analyzes of antibiotic residue detection in milk were performed following the instructions in the Cow Side II Test kit. Of the 90 samples studied, $63.33 \%$ were positive for the presence of antibiotics, where $70 \%$ of the fresh milk samples, $73.33 \%$ of the pasteurized and $46.67 \%$ of the UHT milk samples showed positive reaction to the presence of antibiotics. antibiotics. It was concluded that in the majority of the samples studied there was the presence of antibiotic residues and the present residues were not quantified to compare with the acceptable limit due to the qualitative kit used for the tests. It should also be noted that despite the non-quantification, the presence of these residues should still be considered a concern for consumers, as it is known that they can cause allergic reactions in individuals with hypersensitivity, as well as exposing consumers to other serious risks.

K e y w o r d s : antibiotics, milk, resistance.

\section{IN TRODUÇÃ O}

O leite bovino é o produto proveniente da ordenha completa de vacas sadias, alimentadas de forma apropriada e em condições sanitárias adequadas (BRASIL, 2011). Sua composição envolve uma variedade de substâncias, como por exemplo, a lactose, glicerídeos, sais minerais, vitaminas, enzimas e proteínas (Pereda, 2005). É um alimento considerado essencial para a dieta humana, portanto, deve apresentar adequadas condições sanitárias, mínima contaminação microbiana e ausência de contaminantes químicos (Vieira et al., 2012).

A constituição química do leite favorece o crescimento microbiano, sendo alguns destes microrganismos benéficos para o consumo humano, no entanto, a atividade microbiana sem controle é prejudicial, pois altera suas propriedades tornando-o impróprio para o consumo (Pereda, 2005).

Além do crescimento microbiano, fatores tais como a raça, alimentação, idade do animal, estágio de lactação ou ainda infecções 
podem alterar a composição química do leite (Nascimento et al., 2001). Dentre as infecções as quais o animal pode apresentar, a mastite é uma das mais comuns. Segundo Tozzetti et al. (2008), a mastite é um processo inflamatório da glândula mamária causada por diversos fatores, sendo $90 \%$ dos casos por bactérias. O impacto da mastite no leite produzido pelo animal causa alterações na composição do mesmo, onde há redução de cálcio, fósforo, gordura e proteína, bem como o aumento de cloro e sódio.

A mastite é a principal causa da aflição das propriedades leiteiras, causando grandes prejuízos (Lopes et al., 2013), onde o impacto da doença nesse contexto se deve à redução da produção de leite, à redução do valor comercial dos animais que possuem a doença e aos gastos com os medicamentos necessários para o tratamento (Costa et al., 2013). O principal método para tratar a doença é o uso de antibióticos. Embora as drogas antimicrobianas sejam fundamentais para combater essas infecções, a utilização das mesmas pode trazer consequências indesejáveis tanto para a saúde animal quanto para a saúde humana, sendo uma dessas consequências a excreção pós-terapia de resíduos desses antibióticos em pequenas concentrações no leite (Pereira et al., 2016).

Ainda sobre esse assunto, antes de iniciar a ordenha, o correto é identificar o animal que está em período de lactação e fazendo uso de algum medicamento, identificar a data de administração e o nome do medicamento, dessa forma tornando-se mais fácil respeitar o período de carência, diminuindo então as chances de serem encontrados no leite os resíduos de antibióticos (Cerva, 2013).

Os riscos à saúde humana causados pela presença desses resíduos são representados principalmente pelas reações alérgicas apresentadas em indivíduos com hipersensibilidade a alguns tipos de antibióticos mesmo que em pequenas quantidades, efeitos tóxicos, e até mesmo desequilíbrio da microbiota intestinal (Benetti et al., 2011). O leite contaminado com antimicrobianos pode trazer problemas críticos também para a indústria de laticínios, podendo esses resíduos diminuir ou inibir a atividade fermentadora de alguns tipos de bactérias (Katiani et al., 2009).

Diante do exposto, a pesquisa é justificada pelo fato de que o leite contaminado com resíduos de antibióticos pode ser prejudicial à saúde humana, bem como a indústria de laticínios. Sendo assim, a presente pesquisa envolve a análise de leites comercializados nas cidades de Bela Cruz e Sobral, ambas localizadas no estado do Ceará, avaliando se há presença desses resíduos, pois sabe-se que alguns proprietários responsáveis pelos animais produtores de leite não respeitam o prazo de carência determinado para a ordenha após a administração de antimicrobianos, bem como também há adulteração com a adição 
proposital destas substâncias, sendo estes uns dos fatores que justifica a presença de resíduos no leite.

MATERIAL E MÉTODOS

TIPO DE ESTUDO

Trata-se de um estudo qualitativo, descritivo e experimental no qual foram obtidos dados e analisados parâmetros químicos (MARCONI \& LAKATOS, 2003).

\section{AMOSTRAGEM}

Foram analisadas 90 amostras de leite, sendo 30 in natura, 30 pasteurizadas e 30 UHT. Semanalmente foram estudadas 10 amostras. As amostras in natura foram coletadas em uma fazenda no município de Bela Cruz - CE e, as amostras de leite pasteurizado e UHT foram coletadas em estabelecimentos comerciais da cidade de Sobral - CE. Após a coleta, as amostras foram, imediatamente, transportadas em caixas isotérmicas ao Núcleo de Bioprospecção e Experimentação Molecular Aplicada (NUBEM), localizado no Centro Universitário INTA UNINTA.

O experimento foi realizado no período de abril a junho de 2017 onde os leites UHT e pasteurizado foram escolhidos de lotes diferentes com a finalidade de evitar repetição de resultados. Na tabela 1 podem ser observadas as semanas da realização dos testes, os tipos de leite coletados em cada semana e a quantidade necessária.

\section{IDENTIFICAÇÃO DE RESÍdUOS DE ANTIBIÓTICOS}

Para a realização dos testes foram seguidas as instruções presentes no manual do kit Cow Side II Test, o qual é um teste de detecção de presença de resíduos de antibióticos beta-lactâmicos e de outros grupos em leite (Charm sciences inc, 2016) e, possui o método de inibição microbiana e indicador de $\mathrm{pH}$, que apresenta como indicador o bromocresol púrpura e uma cultura padrão que se baseia no crescimento de Bacillus stearothermophilus. Com a produção de ácido no meio e alteração do pH, é evidenciada através da alteração de cor, portanto, quando há presença de antimicrobianos o microrganismo é impedido de crescer, não ocorrendo a produção de ácido e consequentemente não havendo a alteração da coloração, se mantendo azul (Lage, 2010).

$\mathrm{O}$ kit foi mantido sob refrigeração de $6^{\circ}-10^{\circ} \mathrm{C}$ no NUBEM sendo retirado apenas para uso. Antes da análise das amostras serem 
realizadas, foi padronizado e realizado um procedimento passo-apasso, que consistia em estar paramentado com os Equipamentos de Proteção Individual (EPIs), preparar o banho-maria com um termômetro acoplado para fazer o acompanhamento da temperatura, fazer a lavagem e desinfecção com álcool a 70\% nas embalagens dos leites e da bancada que seria utilizada para a realização dos testes. Os testes foram realizados uma vez por semana, fazendo então o uso de dez tubos do kit contendo a cultura que reage com o leite, sendo os mesmos descartáveis.

Tabela 1. Amostras coletadas para a pesquisa de resíduos de antibióticos em leite.

\begin{tabular}{|c|c|c|}
\hline Semanas & Tipo de leite & $\begin{array}{c}\text { Quantidade de } \\
\text { leite coletado (L) }\end{array}$ \\
\hline $1^{\mathrm{a}}$ Semana & $\begin{array}{c}\text { In natura }(5 \mathrm{~L}) \\
\text { Pasteurizado }(5 \mathrm{~L}),\end{array}$ & $10 \mathrm{~L}$ \\
\hline $2^{\mathrm{a}}$ Semana & $\begin{array}{c}\text { UHT }(5 \mathrm{~L}) \\
\text { In natura }(5 \mathrm{~L})\end{array}$ & $10 \mathrm{~L}$ \\
\hline $3^{a}$ Semana & $\begin{array}{l}\text { Pasteurizado }(5 \mathrm{~L}) \\
\text { In natura }(5 \mathrm{~L})\end{array}$ & $10 \mathrm{~L}$ \\
\hline $4^{\mathrm{a}}$ Semana & $\begin{array}{c}\mathrm{UHT}(5 \mathrm{~L}) \\
\text { In natura }(5 \mathrm{~L})\end{array}$ & $10 \mathrm{~L}$ \\
\hline $5^{\mathrm{a}}$ Semana & $\begin{array}{l}\text { Pasteurizado }(5 \mathrm{~L}) \\
\text { In natura }(5 \mathrm{~L})\end{array}$ & $10 \mathrm{~L}$ \\
\hline $6^{\mathrm{a}}$ Semana & $\begin{array}{c}\mathrm{UHT}(5 \mathrm{~L}) \\
\text { In natura }(5 \mathrm{~L})\end{array}$ & $10 \mathrm{~L}$ \\
\hline $7^{\mathrm{a}}$ Semana & Pasteurizado & $10 \mathrm{~L}$ \\
\hline $8^{a}$ Semana & $\begin{array}{c}\text { UHT (5L) } \\
\text { Pasteurizado (5L) }\end{array}$ & $10 \mathrm{~L}$ \\
\hline $9^{a}$ Semana & UHT & $10 \mathrm{~L}$ \\
\hline
\end{tabular}

Os leites in natura e pasteurizados foram coletados um dia antes das análises, sendo mantidos sob refrigeração entre $6^{\circ}-10^{\circ} \mathrm{C}$ e, os leites UHT foram coletados uma semana antes de serem realizadas as suas respectivas análises.

De acordo com o protocolo presente no kit foram pipetados $100 \mu \mathrm{l}$ de leite para cada um dos dez tubos que foram enumerados de 1 a 90 no total, sendo colocados no banho-maria à temperatura de $64^{\circ} \mathrm{C} \pm 0,5^{\circ} \mathrm{C}$. Após isso esperou-se 3 horas, respeitando o protocolo do manual, para só então realizar a leitura dos resultados. Para obtenção dos resultados foram visualizadas as colorações presentes nos tubos após a interação com o leite. Tubos com a coloração azul/roxa indica que houve uma 
resposta positiva para a presença de resíduos de antibióticos no leite, coloração intermediária, verde petróleo, sugere resultado inconclusivo e, o tubo com coloração verde característica indica que o resultado é negativo para a presença de resíduos.

O Cow Side II Test é um teste que possui seletividade, ou seja, as amostras que não possuem antimicrobianos apresentam 90\% dos resultados negativos com $95 \%$ de confiança e possui sensibilidade aos antimicrobianos detectados como positivos pelo teste, comparando aos níveis regulatórios (Charm sciences inc, 2016).

\section{RESULTADOS E DISCUSSÃO}

\section{PRESENÇA DE RESÍdUOS DE ANTIBIÓtICOS NOS LEITES}

As figuras 1 , 2 e 3 mostram as representações dos resultados obtidos no decorrer do experimento nas amostras de leite estudadas, como positivo, negativo e inconclusivo, respectivamente.
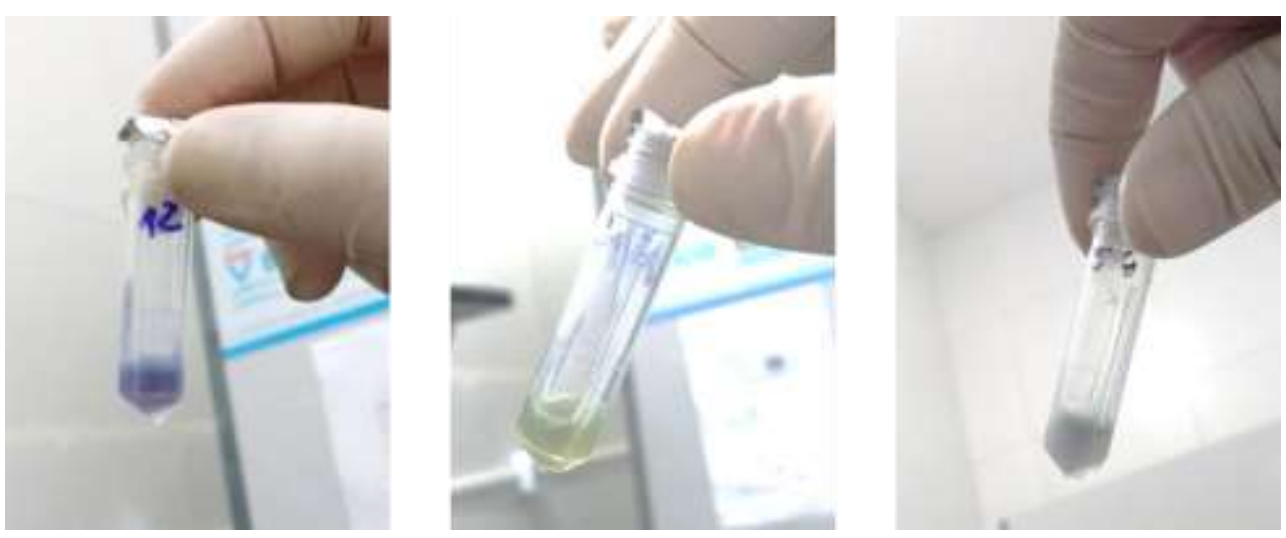

Figura 1. Resultado Positivo. Figura 2. Resultado Negativo. Figura 3. Inconclusivo.

Na figura 4 é possível observar os resultados obtidos nas amostras de leite estudadas. Como pode ser observado, a maioria das amostras apresentou reação positiva, o que indica a presença de resíduos de antibióticos, além disso, algumas amostras também apresentaram resultados inconclusivos, ou seja, não é possível afirmar se há ou não a presença de resíduos de antibióticos. Isso pode ter ocorrido devido a presença fidedigna de resíduos de antibióticos, bem como a presença de outras substâncias no leite ou até mesmo alterações de decomposição que o mesmo possa ter sofrido, impedindo assim resultados satisfatórios. 


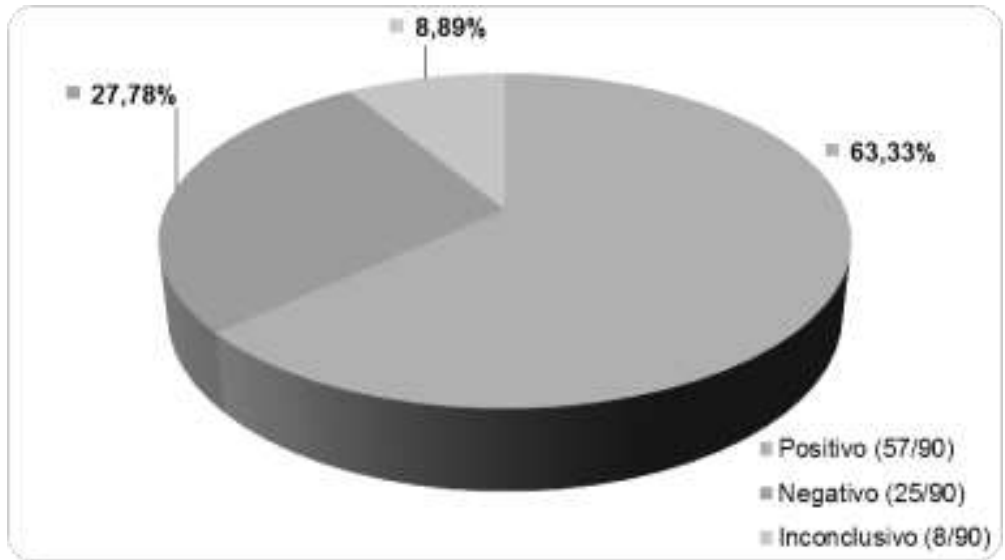

Figura 4. Percentual de amostras de leite com resultados positivos, negativos e inconclusivos para resíduos de antibióticos.

Segundo Horta (2017), testes realizados para a detecção de resíduos de antibióticos podem apresentar falso-positivos e que isso ocorre principalmente em amostras de leite retiradas de gado infectado com mastite, onde o sistema de defesa do animal produz substâncias para combater a infecção. Essas substâncias produzidas são capazes de inibir o crescimento microbiano fazendo com que a amostra seja interpretada erroneamente.

De acordo com o boletim técnico divulgado pela Zoetis (2013), a principal explicação para resultados errôneos ou inconclusivos no leite é a presença de substâncias tais como as lactoferrinas e lisozimas, interferindo assim nos testes e, por isso, é recomendado que os testes sejam realizados em leites colocados em tanques e não em amostras individuais de vacas.

\section{LEITES IN NATURA}

Pode-se observar através da figura 5, que a quantidade de amostras positivas está se sobrepondo a quantidade de amostras com resultado negativo. Isso pode ser justificado pelo uso inadequado de antimicrobianos, onde com o objetivo de ter mais carne e menos gado, os fazendeiros adicionam antibióticos na alimentação do animal para engordá-lo. Pode ser justificado também pelo não respeito ao período de carência após a administração dessas substâncias para o tratamento de infecções ou prevenção das mesmas. 


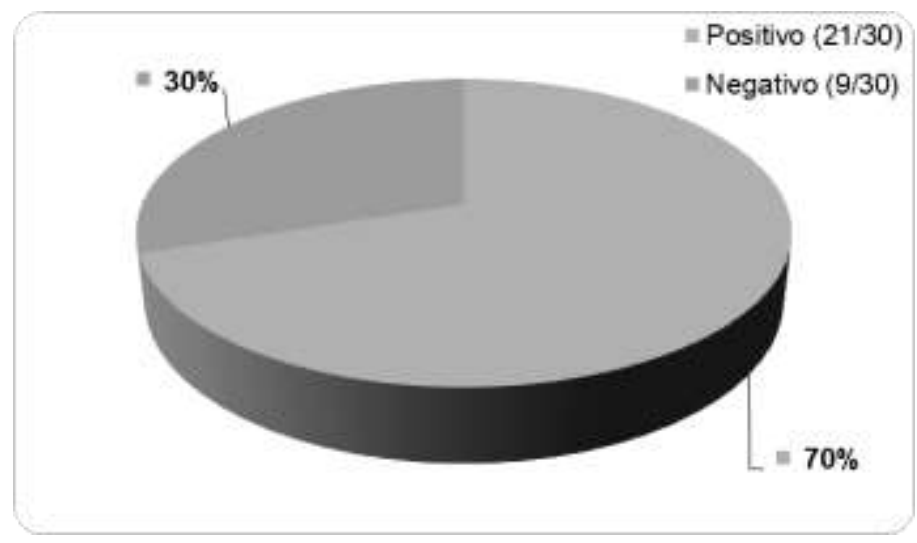

Figura 5. Percentual de amostras de leite in natura com resultados positivos e negativos para a presença de resíduos de antibióticos.

Os resultados positivos para a presença de resíduos de antibióticos foram superiores aos reportados por Araújo et al. (2015), os quais realizaram testes em 235.293 litros de leite in natura na plataforma de recepção de laticínios sob inspeção federal, observando a presença de resíduos de antibióticos em 20 mil litros. Já Souza et al. (2017) analisaram 112 amostras de leite in natura no estado do Rio Grande do Norte e verificaram que $6,72 \%$ das amostras apresentaram resultado positivo para a presença de resíduos de antibióticos. Ferreira et al. (2014), obtiveram $2,33 \%$ de amostras com resultado positivo ao analisarem 86 amostras de leite in natura de um laticínio localizado em Teresina.

Segundo Lima (1994), os antibióticos são administrados no gado para aumentar o ganho de peso, sendo as tetraciclinas as mais utilizadas e, segundo Consolaro (2011), o uso constante de antibióticos na alimentação bovina pode favorecer a criação de superbactérias resistentes, onde essas bactérias sofrem mutações genéticas em seu DNA e transmitem essas mutações para as gerações futuras. Portanto pode-se sugerir que os resíduos de antibióticos presentes no leite podem causar prejuízos a saúde dos consumidores deste alimento.

A prevenção da contaminação do leite por resíduos de antibióticos é de responsabilidade de toda a cadeia produtiva, mas principalmente do veterinário e proprietário do gado leiteiro. Segundo Santos (2015), vacas que estão em lactação e são submetidas a tratamento com antibióticos necessitam de um período de carência do leite, durante o qual toda a produção do bovino leiteiro deve ser descartada, no entanto, muitas vezes esse período não é respeitado ou houve uma utilização inadequada desses antimicrobianos no período pré-parto, ocasionando a presença de resíduos dessas substâncias no leite. 
Na figura 6 é possível verificar o percentual de amostras de leite que apresentaram resíduos de antibióticos.

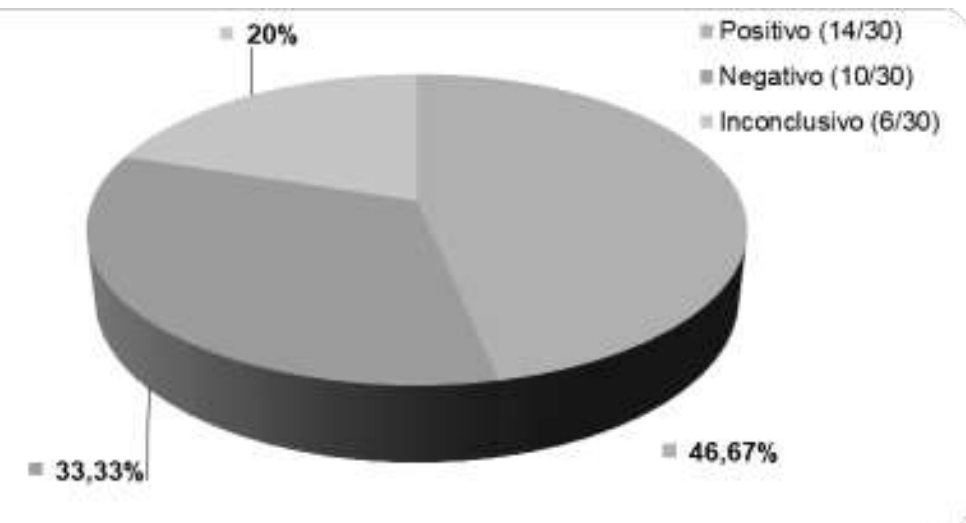

Figura 6. Percentual de amostras de leite pasteurizado com resultados positivos, negativos e inconclusivos para a pesquisa de resíduos de antibióticos.

O percentual de amostras com resultados positivos foi superior a quantidade de amostras sem resíduos de antimicrobianos. Isso pode ter ocorrido devido a adição proposital de antibióticos no leite, caracterizando uma adulteração do produto, pois é sabido que a pasteurização do leite é suficiente para eliminar os microrganismos patogênicos, no entanto existem bactérias que sobrevivem ao processo de pasteurização e que, apesar de não causarem doenças aos consumidores podem estragar o produto em determinadas condições. Segundo Folly \& Machado (2001), a contaminação do leite pasteurizado por resíduos de antibióticos pode ocorrer direta ou indiretamente, podendo ser através da adulteração, com o objetivo de prolongar a vida útil do leite ou por consequência de tratamentos indicados para o gado leiteiro.

O presente estudo apresentou resultados semelhantes aos de Sousa et al. (2010), os quais realizaram pesquisa de resíduos de antibióticos em 30 amostras de leite pasteurizado, oriundas do Cariri - CE e, 73,67\% das amostras estudadas apresentaram resultado positivo para a presença de resíduos de antibióticos. Porém, divergiram dos resultados encontrados por Folly \& Machado (2001), os quais estudaram 300 amostras de leites pasteurizados comercializados na região norte do Rio de Janeiro e concluíram que 13 amostras apresentaram presença de resíduos de antimicrobianos e, divergiram também dos resultados encontrados por Magnetiva (2012), onde das 240 amostras de leite pasteurizado coletadas das regiões sudoeste e sul bahiano, apenas 7,9\% mostraram-se positivas.

Os resultados obtidos no presente estudo mostraram-se divergentes também da pesquisa realizada por Brito et al. (2016), onde após analisar 100 amostras de leites pasteurizados oriundos de Ubatuna - BA, obteve 
como resultado $27 \%$ de amostras positivas para a presença de resíduos de antibióticos.

\section{LEITES UHT}

A figura 7 mostra o percentual de amostras com resultados positivos, negativos e inconclusivos para a presença de resíduos de antibióticos em leite UHT.

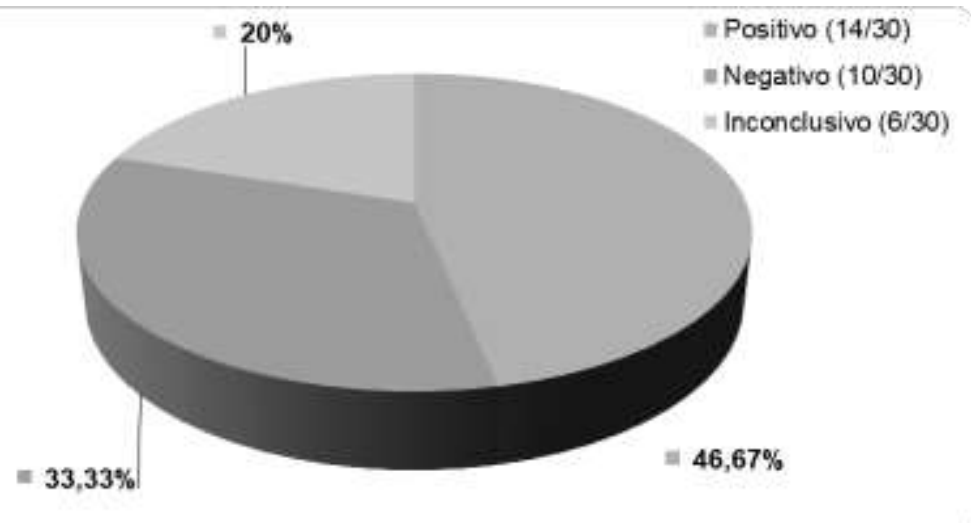

Figura 7. Percentual de amostras de leite UHT com resultados positivos, negativos e inconclusivos para a presença de resíduos de antibióticos.

Pode-se observar que a porcentagem de amostras positivas predominou, no entanto, observa-se também que a porcentagem de amostras negativas foi elevada e amostras inconclusivas ficaram a meio termo. A positividade das amostras pode ser justificada pela adição proposital de antibióticos com o intuito de prolongar a vida útil do leite ou pelo não controle adequado do leite utilizado para a produção.

A grande quantidade de amostras negativas pode ser justificada pelo fato do leite UHT sofrer processos mais rigorosos e uma fiscalização interna da indústria responsável mais efetiva comparada aos leites in natura e pasteurizado, mas ainda sujeita a erros. Por ser um dos tipos de leite mais consumidos mundialmente, seguido do pasteurizado, deve haver uma maior preocupação com sua qualidade, garantindo um produto em bom estado e que não tragam riscos à saúde do consumidor.

Apesar de ter sido o tipo de leite que apresentou maior percentual de resultados negativos para a presença de resíduos de antibióticos, ainda apresentaram valores elevados quando comparado ao estudo realizado por Costa \& Lobato (2009), os quais analisaram 175 amostras de leites UHT comercializados em Seropédica - RJ, utilizando o kit Devoltest e, apenas 2 apresentaram resultados positivos para a presença de resíduos de antibióticos. Silva (2011) realizou testes também utilizando com o kit Devoltest em 224 amostras de leite UHT coletadas no estado de 
Goiás e, obteve como resultado, 12 amostras positivas e 212 negativas para a presença de resíduos de antibióticos em leite UHT.

Os resultados obtidos nesse estudo também divergiram dos apresentados por Fonseca et al. (2009), que ao realizarem análise de 100 amostras de leite UHT em várias regiões do Brasil, apenas 4 mostraramse positivas para a presença de resíduos de antimicrobianos.

\section{CONCLUSÃO}

Os testes realizados não informaram especificamente qual o antibiótico e nem a concentração encontrada por ser de caráter qualitativo, no entanto, 57 amostras (63,33\%) se apresentaram positivas para beta-lactâmicos e outros grupos não definidos. Essa informação é importante, porque dentre os antibióticos administrados em gado leiteiro, os beta-lactâmicos são considerados um dos mais utilizados, além de ser a classe que mais causa reações de hipersensibilidade em pessoas alérgicas, devendo então os produtores de leite e indústrias fiscalizarem mais rigorosamente os leites que estão sendo produzidos e colocados no mercado.

Apesar de ser aceita uma concentração mínima padronizada de substâncias em alimentos, deve-se pensar que, em pessoas que possuem alta susceptibilidade de desenvolver reações alérgicas, uma quantidade mesmo que mínima não impede o desenvolvimento das mesmas. E sabe-se também que há frequentemente adulteração no leite, uso descontrolado de antimicrobianos e o não respeito do período de carência e por isso não se pode dizer à risca que os leites analisados estavam respeitando a quantidade mínima padronizada, sendo todos esses fatores justificativas para a presença de resíduos dessas substâncias no leite.

Dentre os três tipos de leite analisados, pode-se concluir que os leites pasteurizados foram os que apresentaram mais resultados positivos e isso é preocupante para a saúde dos consumidores por ser um dos leites mais consumidos no Brasil juntamente com o leite UHT, levando riscos à saúde que varia desde o desequilíbrio da microbiota intestinal até efeitos mais graves, que atingem a medula óssea.

\section{REFERÊNCIAS}

Araújo, G. B., Santos, H. A., Farias, C. E., Viana, D. A. F., Vieira, E. S., \& Júnior, A. M. F. (2015). Detecção de resíduos de antibióticos em leite in natura em laticínio sob inspeção fereral. Scientia Plena, 11(4), 1-6.

Benetti, T. M., Abrahão, W. M., \& Nickel, R. (2011). Análise comparativa entre os ensaios imunoenzimáticos e microbiológicos para a detecção de resíduos de 
antibióticos em leite. Revista do Instituto de Laticínios Cândido Tostes, 66(381), 41-45.

Brasil. Instrução Normativa $n^{o} 62$ de 29 de dezembro de 2011. (2011). Ministério da Agricultura, Pecuária e Abastecimento. Diário Oficial da República Federativa do Brasil. Recuperado de http://www.apcbrh.com.br/files/IN62.pdf.

Brito, U. M. B., Souza, L, M., Leite, P. A. G., \& Ribeiro, A. R. P. (2016). Detecção de resíduos de substâncias com ação antimicrobiana nos leites UAT e pasteurizado comercializados no município de Itabuna - Bahia. Veterinária e Zootecnia, 23(1), 123-130.

Cerva, C. (2013). Manual de Boas práticas na produção de leite em propriedades de agricultura familiar do Rio Grande do Sul. Porto Alegre: Fepagro. Recuperado de http://www.fepagro.rs.gov.br/upload/20130730144014manual_boas_praticas_1 eite.pdf.

Charm Sciences Inc. (2016). Charm cowside II test for beta-lactams and other antimicrobial drugs in milk. Recuperado de https://www.charm.com/products/test-and-kits/antibiotic-tests/inhibitiontests/charm-cowside-ii-test/.

Consolaro, A. (2011). A carne bovina, receita médica e superbactérias. Recuperado de http://www.jcnet.com.br/Ciencias/2011/01/carne-bovina-receita-medica-esuperbacterias.html.

Costa, A. S., \& Lobato, V. (2009). Avaliação da presença de resíduos de antimicrobianos em leite e bebida láctea UHT por teste de inibição microbiana comercial. Revista do Instituto de Laticínios Cândido Tostes, 64(368), 72-76.

Costa, G. M., Rodrigo, A. B., Custódio, D. A. C., Pereira, U. P., Figueiredo, D. J., \& Silva, N. (2013). Resistência a antimicrobianos em Staphylococcus aureus isolados de mastite em bovinos leiteiros de Minas Gerais, Brasil. Arquivos do Instituto Biológico, 80(3), 297-302.

Ferreira, A. R. P., Lívia, S. O. M., Rocha, T. S., \& Ramos, L. S. N. (2014). Resíduos de antibióticos em leite in natura utilizado para processamento em laticínio localizado no município de Teresina - Piauí. Acta Tecnológica, 9(1), 9-12.

Folly, M. M., \& Machado, S. C. A. (2001). Determinação de resíduos de antibióticos, utilizando-se métodos de inibição microbiana, enzimático e imuno-ensaios no leite pasteurizado comercializado na região norte do estado do Rio de Janeiro, Brasil. Ciência Rural, 31(1), 95-98.

Fonseca, G. P., Cruz, A. G., Faria, J. A. F., Silva, R., Moura, M. R. L., \& Carvalho, L. M. J. (2009). Antibiotic residues in brazilian UHT milk: a screening study. Ciência e Tecnologia de Alimentos, 29(2), 451-453.

Horta, M. Métodos diagnósticos de resíduos de antibióticos no leite. (2017). Recuperado http://m.milkpoint.com.br/mypoint/404548/p_metodos_diagnosticos_de_residu os_de_antibioticos_no_leite_leite_antibiotico_antibiotico_no_leite_residuos_no_l eite_6255.aspx.

Katiani, L., Farre, M., \& Barcelo, D. (2009). Analytical methodologies for the detection of b-lactam antibiotics in milk and feed samples. Trends in Analytical Chemistry, 28(6), 729-744. 
Lage, A. D. (2010). Avaliação do charm cow side II test e charm blue yellow II test para a detecção de resíduos de antimicrobianos em leite (Dissertação de mestrado). Departamento de Tecnologia e Inspeção de produtos de origem animal, Universidade de Minas Gerais, Belo Horizonte, Brasil.

Lima, J. O. A. A. (1994). Nutrição do bovino de corte confinado. Recuperado de https://www.embrapa.br/busca-de-publicacoes/-/publicacao/356172/nutricaodo-bovino-de-corte-confinado.

Lopes, L. O., Lacerda, M. S., \& Ronda, J. B. (2013). Uso de antibióticos na cura e controle de mastite clínica e subclínica causada por principais microorganismos contagiosos em bovinos leiteiros: Revisão de literatura. Recuperado de http://faef.revista.inf.br/imagens_arquivos/arquivos_destaque/RyKnT9CEMC8Q 2de_2013-8-13-18-19-12.pdf.

Magnetiva, A. P. A. (2012). Avaliação das características físico-químicas e da presença de resíduos de antimicrobianos em leite pasteurizado nas regiões sudoeste $e$ sul bahiano. Recuperado de http://www2.uesb.br/ppg/ppgecal/wpcontent/uploads/2017/04/ANA-PRUDENCIA-MAGNAVITA.pdf.

Marconi, M. A., \& Lakatos, E. M. (2003). Fundamentos da metodologia científica. 5ed. São Paulo: Editora Atlas.

Nascimento, G. G. F., Maestro, V., \& Campos, M. S. P. (2001). Ocorrência de resíduos de antibióticos no leiro comercializado em Piracicaba, SP. Revista Nutrição, 14(2), 119-124.

Pereda, J. A. O. (2005). Tecnologia de alimentos - Alimentos de origem animal. Porto Alegre, RS: Editora Artmed.

Pereira, R. V. V., Lima, S., Siler, J. D., Foditsch, C., Warnick, L. D., \& Bicalho, R. C. (2016). Ingestion of milk containing very low concentration of antimicrobials: longitudinal effect on fecal microbiota composition in preweaned calves. Plos one, 11(1), 1-18.

Santos, M. V. (2015). Controle e prevenção de resíduos no leite. Recuperado de http://qualileite.org/pdf/Artigos-tecnicos-publicados-em-revista-dedivulgacao/Inforleite/2015/1-2015-Inforleite-Controle-de-residuos-deantibioticos.pdf.

Silva, T. S. (2011). Qualidade do leite produzido no estado de Goiás - ocorrência de resíduos de antimicrobianos e acidez titulável (Dissertação de mestrado). Departamento de Sanidade Animal, Higiene e Tecnologia de Alimentos, Universidade Federal de Goiás, Goiânia, Brasil. Recuperado de https://ppgca.evz.ufg.br/up/67/o/Dissertacao2011_Thaysa_Santos.pdf.

Sousa, F. C., Oliveira, E. N. A., Santos, D. C., \& Silva, E. F. M. (2010). Ocorrência de resíduos de antibióticos em leites pasteurizados comercializados no estado do Ceará - Brasil. Revista Verde, 4(4), 10-14.

Souza, L. B., Pinheiro, C. G. M. E., Neto, S. A. G., \& Silva, J. B. A. (2017). Resíduos de antimicrobianos em leite bovino cru no estado do Rio Grande do Norte. Recuperado de https://revistas.ufg.br/vet/article/view/e-23050/24510.

Tozzeti, D. S., Bataier, M. B. N., \& Almeida, L. R. (2008). Prevenção, controle e tratamento das mastites bovinas. Recuperado de http://faef.revista.inf.br/imagens_arquivos/arquivos_destaque/YFbjMNrGCotOL 73_2013-5-28-15-25-40.pdf. 
Vieira, T. S. W. J., Ribeiro, M. R., Nunes, M. P., Júnior, M. M., \& Netto, D. P. (2012). Detecção de resíduos de antibióticos em amostras de leite pasteurizado do Estado do Paraná Brasil. Semina: Ciências Agrárias, 33(2), 791-796.

Zoetis. (2013). Resíduos de produtos antimicrobianos: descarte zero x resíduos zero. Recuperado http://187.108.194.18/ resolpec/UPLarquivos/0502201408201214.pdf. de

\section{MINICURRÍCULO}

ANA BEATRIZ MELO GUIMARÃES Possui graduação em Farmácia e pósgraduação em Farmácia Clínica e Prescrições Farmacêuticas.

MÁRCIA FACUNDO ARAGÃo Possui graduação em Engenharia de Alimentos pela Universidade Federal do Ceará e mestrado em Ciência e Tecnologia dos Alimentos pela Universidade Federal do Ceará. curso de graduação em Biomedicina, Instituto Esperança de Ensino Superior (IESPES), Santarém-PA Brasil.

RENATA ALBUQUerQue COSTA Possui graduação em Licenciatura em Biologia pela Universidade Estadual Vale do Acaraú, mestrado em Ciências Marinhas Tropicais pela Universidade Federal do Ceará e doutorado em Engenharia de Pesca.

JOSÉ RODOLFO TEIXEIRA DA CUNHA Possui graduação em Enfermagem pela Universidade Estadual Vale do Acaraú. Graduando em Medicina pela Universidade Federal do Maranhão.

THAIANE VASCONCELOS CARVALHO Possui graduação em Farmácia e pós-graduação em Farmácia Clínica e Prescrições Farmacêuticas. 